\title{
Fermi Gap Stabilization of an Incommensurate Two-Dimensional Superstructure
}

\author{
F. Schiller, ${ }^{1,2}$ J. Cordón, ${ }^{3}$ D. Vyalikh, ${ }^{2}$ A. Rubio, ${ }^{1,4,5}$ and J. E. Ortega ${ }^{1,3,4}$ \\ ${ }^{1}$ Donostia International Physics Center, Paseo Manuel Lardizabal 4, E-20018 Donostia-San Sebastián, Spain \\ ${ }^{2}$ Institut für Festkörperphysik, TU Dresden, D-01062 Dresden, Germany \\ ${ }^{3}$ Departmento Física Aplicada I, Universidad del País Vasco, E-20018 Donostia-San Sebastián, Spain \\ ${ }^{4}$ Unidad de Física de Materiales CSIC/UPV, Manuel Lardizabal 3, E-20018 Donostia-San Sebastián, Spain \\ ${ }^{5}$ Departmento Física Materiales, Universidad del País Vasco, E-20018 Donostia-San Sebastián, Spain
}

(Received 10 September 2004; published 6 January 2005)

\begin{abstract}
The compressed, incommensurate $\sim(9.5 \times 9.5)$ moiré superstructure of the Ag monolayer on $\mathrm{Cu}(111)$ displays a filled surface state band with a Fermi energy gap at the Brillouin zone boundary. By contrast, the surface band is gapless for the less compressed, commensurate $(9 \times 9)$ moire of two Ag layers. A simple estimate of the energy gain rendered by opening this gap gives a value similar to the elastic energy change required to modify the commensurate structure, thereby suggesting that the $\sim(9.5 \times 9.5)$ incommensurate phase is stabilized by such a gap opening. The possible presence of a charge density wave state is discussed.
\end{abstract}

DOI: 10.1103/PhysRevLett.94.016103

Nanostructures grown on solid surfaces are of fundamental importance for future device technology. They exhibit electronic and magnetic properties due to their reduced dimensions. New phases appear that do not have a bulk counterpart, e.g., incommensurate phases, which can drive structural phase transitions. Incommensurability is particularly important in the context of cooperative phenomena like superconductivity [1], or spin and charge density wave transitions [2,3]. In the latter, an incommensurate phase can result from the nesting of the Fermi surface that defines the new lattice constant, which in turn allows a band gap to open up at the Brillouin zone boundary and at the Fermi energy. This lowers the electron energy, balancing the cost to build up the incommensurate phase. In two-dimensional (2D) systems electron energy changes due to band gap openings are relatively small, and hence structural transitions associated with electron band changes are very scarce [4]. In this Letter we provide clear evidence for an electronically driven stabilization of a 2D incommensurate phase, namely, the $\sim(9.5 \times 9.5) \mathrm{Ag}$ monolayer on $\mathrm{Cu}(111)$.

In thermal equilibrium, crystal growth is driven by free energy minimization, i.e., the balance between surface energy and the stress energy accumulated at the interface. The latter is originated by the substrate-overlayer lattice mismatch that forces the growing layer to compress or expand in order to adopt a minimum energy configuration. In some cases, the stress energy at the interface is relatively large with respect to the surface energy, and the system breaks into two- or three-dimensional islands. In the layerby-layer growth mode the surface energy of the growing material is very low, and the overlayer smoothly recovers its bulk crystal structure as it becomes thicker. The $1 \mathrm{ML}$ (monolayer) $\mathrm{Ag} / \mathrm{Cu}(111)$ system is an interesting example of layer-by-layer growth in large $(13 \%)$ mismatched materials. In this case, the Ag monolayer wets the substrate, forming a compressed, out-of-registry $\sim(9.5 \times 9.5)$ moiré
PACS numbers: 68.35. $-\mathrm{p}, 73.20 .-\mathrm{r}$

superstructure, with a lattice compression of $\sim 1.1 \%$ with respect to the bulk $\operatorname{Ag}(111)$ plane $[5,6]$. The question arises why the system favors in this case the out-of-registry $\sim(9.5 \times 9.5)$ structure instead of, for instance, the registry $(9 \times 9)$, with only $0.4 \%$ lattice compression. Here we show that this is likely due to the surface electronic structure. Indeed, the Fermi gap is observed only in the surface band of the $\sim(9.5 \times 9.5) \mathrm{Ag}$ monolayer, in contrast with the gapless surface band that characterizes the registry $(9 \times 9)$ superstructure of the two Ag layer film. An estimate of the electron energy gain caused by the gap opening is made and shown to be compatible with the elastic energy needed to modify the $(9 \times 9)$ registry reconstruction.

The photoemission experiments were performed at $150 \mathrm{~K}$ with a Scienta 200 high-resolution angle resolved hemispherical analyzer. The sample was illuminated with monochromatized photons of $h \nu=21.2 \mathrm{eV}$. Energy and angular resolutions were set to $30 \mathrm{meV}$ and $0.3^{\circ}$, respectively. Ag is deposited on top of a $\mathrm{Cu}(111)$ single crystal held at $150 \mathrm{~K}$ and shortly annealed to $300 \mathrm{~K}$. This procedure leads to a triangular structure with $\mathrm{Cu}$ vacancies and dislocation loops in the substrate [5,7]. Scanning tunneling microscopy (STM) was carried out in a different chamber and images were taken at $300 \mathrm{~K}$. Figure 1(a) shows the STM topography for a 0.4 ML Ag film. Very large, monolayer thick $\mathrm{Ag}$ islands with the characteristic moiré reconstruction can be observed. The low energy electron diffraction (LEED) pattern reveals a hexagonal $\sim(9.5 \times$ 9.5) ordered structure with a slight $2.5^{\circ}$ azimuthal rotation with respect to the $\mathrm{Cu}$ substrate, as reported previously [5]. In order to have an estimate of the nearest neighbor separation in the compressed $\operatorname{Ag}(111)$ overlayer, we may assume that the size mismatch homogeneously accommodates along the close-packed $\langle 110\rangle$ directions of the $\mathrm{Cu}$ substrate. Thereby the $(n \times n)$ reconstruction leads to a $\delta=1-\left(a_{\mathrm{Cu}} / a_{\mathrm{Ag}}\right) \times[n /(n-1)]$ lattice compression, with $a_{\mathrm{Cu}}=3.615 \AA$ and $a_{\mathrm{Ag}}=4.085 \AA$ being the 


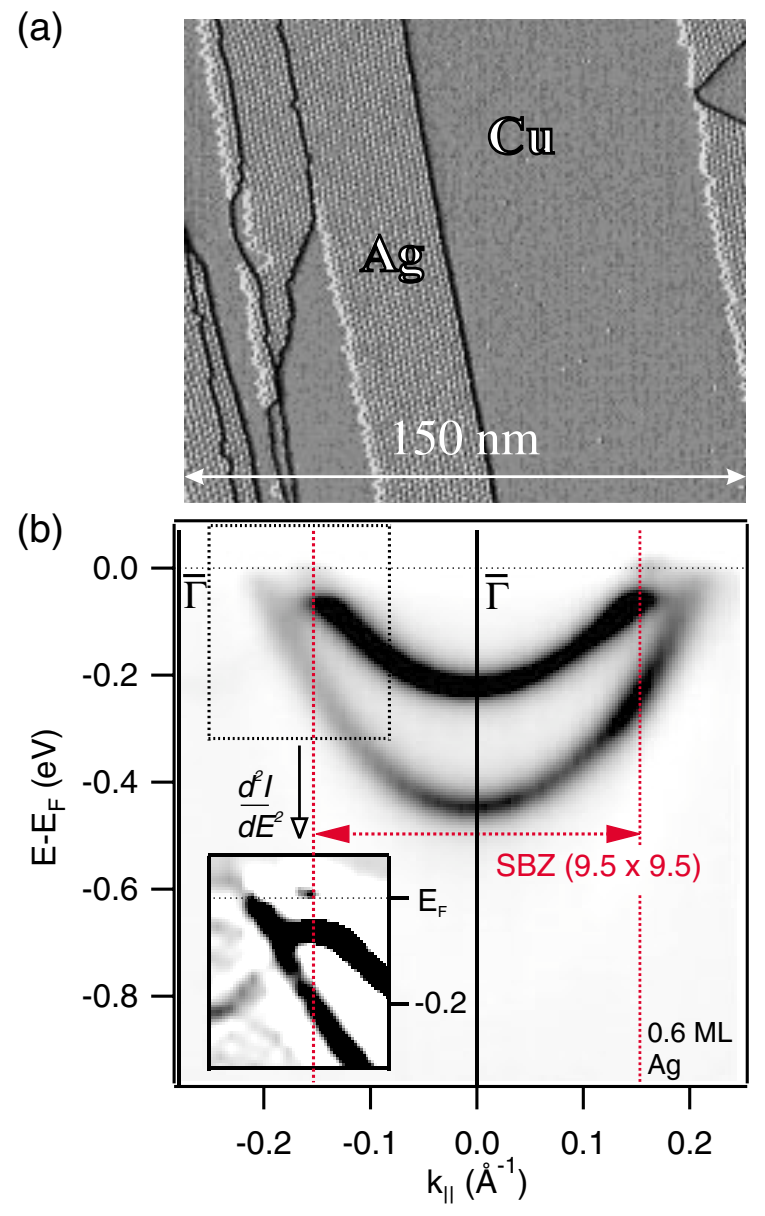

FIG. 1 (color online). (a) STM topography for $0.4 \mathrm{ML}$ of $\mathrm{Ag}$ on $\mathrm{Cu}(111)$ that shows its characteristic moiré. Large, $1 \mathrm{ML}$ thick $\mathrm{Ag}$ covered patches and clean $\mathrm{Cu}$ areas lead to distinct surface bands in (b). Photoemission intensity image for 0.6 ML $\mathrm{Ag} / \mathrm{Cu}(111)$. The inset shows the second derivative spectra around the $\sim(9.5 \times 9.5)$ superstructure zone boundary, where the $1 \mathrm{ML}$ Ag band folds back, opening a gap up to the Fermi energy. The tiny intensity enhancement above the Fermi energy at the zone boundary corresponds to the upper edge of the energy gap, visible by thermal broadening.

respective bulk lattice constants of $\mathrm{Cu}$ and $\mathrm{Ag}$. Hence the $\sim(9.5 \times 9.5)$ reconstruction corresponds to a $\operatorname{Ag}(111)$ close-packed layer with $\delta=1.1 \%$ lattice compression [8].

Figure 1(b) shows a photoemission image of the $p_{z}$-like surface state dispersion along [110] for a 0.6 ML Ag film. As pointed out previously by Bendounan et al. [9], we can observe a double band due to incoherent emission from clean $\mathrm{Cu}(111)$ patches (band with minimum at $E_{\mathrm{Cu}}=$ $E_{F}-435 \mathrm{meV}$ ) and from $1 \mathrm{ML}$ thick $\mathrm{Ag}$ islands (minimum at $E_{1 \mathrm{ML} \mathrm{Ag}}=E_{F}-217 \mathrm{meV}$ ). The inset shows the second derivative of the spectra around the surface Brillouin zone (SBZ) boundary for a better visualization of the band topology. There is a striking difference between $\mathrm{Ag}$ and $\mathrm{Cu}$ bands. The $\mathrm{Cu}$ band clearly displays Fermi level crossing, whereas the $\mathrm{Ag}$ band backfolds at $0.15 \AA^{-1}$, i.e., at the SBZ boundary of the $\sim(9.5 \times 9.5)$ moire structure (marked with vertical dotted lines), leading to a clear SBZ gap that opens up at the Fermi energy.

Figure 2 shows the surface state evolution from 1 to $2 \mathrm{ML}$. For $2 \mathrm{ML}$ the surface band is shifted to $E_{F}-$ $165 \mathrm{meV}$, in agreement with previous observations [9]. The $2 \mathrm{ML}$ band displays zone folding as in the $1 \mathrm{ML}$ case, but the surface zone boundary increases to $0.16 \AA^{-1}$, which corresponds to half of the reciprocal lattice vector for a $(9 \times 9)$ moiré reconstruction. This agrees with STM and LEED that display a $(9 \times 9)$ pattern. Thus the lattice compression with respect to bulk $\mathrm{Ag}(111)$ in the $2 \mathrm{ML}$ surface is reduced to $0.4 \%$, and the system has recovered a registry reconstruction.

By contrast with the $1 \mathrm{ML}$ band, the $2 \mathrm{ML}$ band does not show any gap opening at $E_{F}$. Gap values are quantitatively analyzed in Fig. 3. Features that lie within $\pm 2 k_{B} T$ of $E_{F}$ are cut off by the Fermi edge. Peaks above $E_{F}$ can thus be visualized by normalizing the energy distribution curves

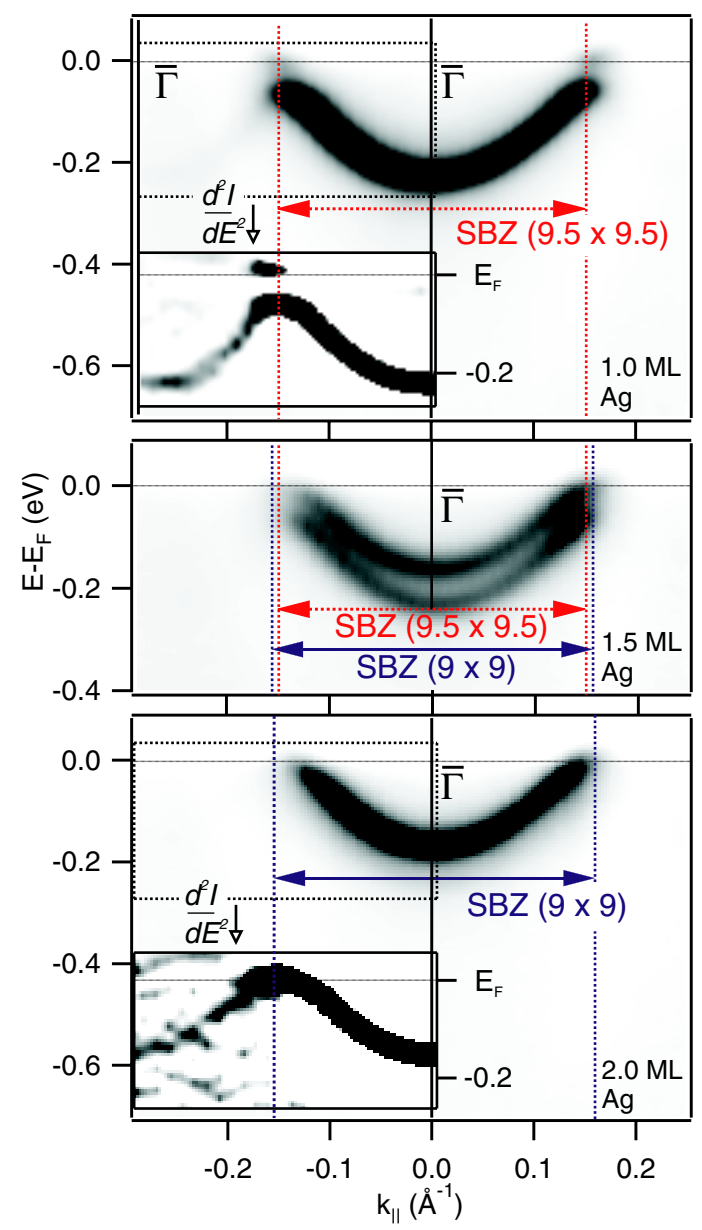

FIG. 2 (color online). Evolution of surface bands from 1 to 2 ML. The Fermi band gap vanishes for $2 \mathrm{ML}$, although a band folding shifted towards the $(9 \times 9)$ superstructure zone boundary can still be observed. For the 1 and $2 \mathrm{ML} \mathrm{Ag} / \mathrm{Cu}(111)$ the insets show the second derivative spectra that enhance the peak features. 
(EDC's) at the SBZ with the Fermi-Dirac distribution function convoluted with the experimental resolution [10]. Indeed, for 0.6 and 1 ML two Ag peaks may be observed, one above and one below the Fermi energy, which, respectively, correspond to the upper and lower band gap edges. For the $2 \mathrm{ML} \mathrm{Ag} / \mathrm{Cu}(111)$ case the surface state crosses clearly the Fermi energy, and hence the peak above $E_{F}$ corresponds to the lower edge of the gap. The accurate position of the band gap edges is obtained from a standard line fit of the photoemission spectra in Fig. 3 using Lorentzian functions for the photoemission peaks, convoluted with Gaussian lines and Fermi edge functions [11]. The lower edges of the zone-boundary gaps are found at $-(65 \pm 5)$ and $+(17 \pm 5) \mathrm{meV}$ for 1 and $2 \mathrm{ML}$, respectively. The upper edge of the gap for $1 \mathrm{ML}$ is found at $+(21 \pm 5) \mathrm{meV}$. Thus for $1 \mathrm{ML}$ the zone-boundary gap amounts to $\Delta=(86 \pm 10) \mathrm{meV}$ and straddles the Fermi level, whereas for $2 \mathrm{ML}$ any SBZ gap would be fully located above $E_{F}$.

In a two-band model, the Fourier component of the electronic potential $V_{G}$ that opens the zone-boundary gap $\Delta$ is given by $V_{G}=\Delta / 2$. From the experimental data in Fig. 3 we get $V_{G}=43 \mathrm{meV}$. This value is consistent with the variation of the effective electron potential induced by a periodic modulation of the silver monolayer along the [110] direction, i.e., following the $(9.5 \times 9.5)$ moiré with a vertical $2 \Delta z=0.3 \AA$ modulation [5]. Indeed, we can estimate the interaction energy of this modulation within the harmonic approximation for the deformation potential. The relevant phonon frequencies for that motion lie between 12 and $20 \mathrm{meV}$ [12]. Considering an average value for the phonon frequency of $\hbar \tilde{\omega}=16 \mathrm{meV}$, and an average atom displacement of $\Delta z=0.15 \AA$, the estimated interaction energy is $E=(1 / 2) M_{\mathrm{Ag}} \Delta z^{2} \tilde{\omega}^{2}=74 \mathrm{meV}$, with $M_{\mathrm{Ag}}$ being the mass of a $\mathrm{Ag}$ atom. If we assume that the major contribution to this energy comes from the interaction between the surface state charge in a fully occupied band $\left(n_{e}^{G} \sim 2\right.$; see below) and the periodic moiré potential $\Delta V=V_{G} e^{i G r}$, then $E \sim \int \Delta V \times n_{e}=V_{G} \times n_{e}^{G}$, and hence we obtain an upper limit estimation for $V_{G}=$ $37 \mathrm{meV}$.

Next we test whether the surface energy gain obtained by opening the Fermi gap is compatible with the extra elastic deformation that is required to modify the overlayer reconstruction. Early tight binding calculations for pseudomorphic $\mathrm{Ag}$ monolayer moirés on $\mathrm{Cu}(111)$ indicate slight changes in surface energy around a $(9 \times 9)$ equilibrium structure [13]. The surface energy per $\mathrm{Ag}$ atom is found to change only within $\Delta U=4 \mathrm{meV}$ when compressing the Ag layer from the $(8 \times 8)$ structure up to the $(10 \times 10)$ one, despite the large $\delta=1.7 \%$ lattice compression in the latter. This suggests that, although a commensurate reconstruction can be expected as the equilibrium configuration (and, indeed, observed for the 2 ML band in Fig. 2), a nearby, intermediate incommensurate reconstruction would suppose only a minor elastic energy increase.

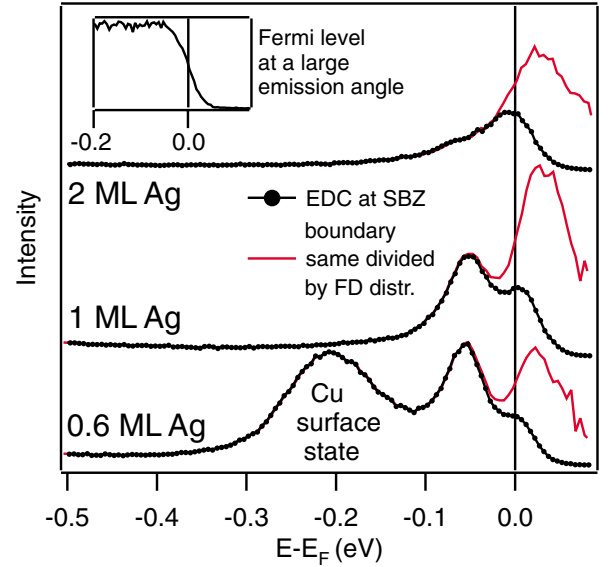

FIG. 3 (color online). Analysis of the band gap for EDC's at the SBZ boundary. Shown are the as-measured EDC's and the same spectra normalized by the Fermi-Dirac distribution function. Inset: EDC taken at a large emission angle defining the Fermi energy.

Furthermore, from the point of view of the lattice compression, since a minor change in the lattice parameter is involved, the change $\Delta U$ from the commensurate $(9 \times 9)$ to the incommensurate $\sim(9.5 \times 9.5)$ could be much less than $4 \mathrm{meV}$. A crude estimate upper bound can be obtained by comparing the elastic energy needed to compress (111) planes in a $\mathrm{Ag}$ crystal. Such energy is given by the equation $U=\frac{1}{2}\left(C_{11}+C_{12}+C_{44}\right) \delta^{2} V_{0}$, where $C_{11}, C_{12}$, and $C_{44}$ are the elastic constants of $\mathrm{Ag}$ [14], $\delta$ the lattice compression in the (111) plane, and $V_{0}$ the bulk volume of silver $\frac{1}{4} a_{\mathrm{Ag}}^{3}$. Assuming a change in compression from $\delta=0.4 \%$ $(9 \times 9$ superstructure) to $\delta=1.1 \%(9.5 \times 9.5)$, we obtain $\Delta U=1.4 \mathrm{meV}$. Actually, $V_{0}$ should be reduced in the $\mathrm{Ag}$ monolayer, since the plane distance $d$ in the vertical direction is rather an arbitrary value between 0 and the plane distance $a_{\mathrm{Ag}} / \sqrt{3}$ in a bulk Ag crystal. Taking $d$ as the metal atomic radius of $\mathrm{Ag}$ to $d=1.42 \AA$ [14], one obtains an estimate for the change in elastic energy from the $(9 \times 9)$ to the $(9.5 \times 9.5)$ structures of $\Delta U=0.48 \mathrm{meV}$ [15].

The increase $\Delta U$ in elastic energy should be balanced by the electronic energy gain of the $\sim(9.5 \times 9.5)$ reconstruction. The gain in surface energy produced by the gap opening in the $1 \mathrm{ML}$ surface band can be estimated from the difference in the electron energy in the $2 \mathrm{D}$ band with and without the zone-boundary gap, as shown in Fig. 4. In both cases we assume the effective mass $m^{*}=0.41 m_{e}\left(m_{e}\right.$ being the electron mass), which is measured around the zone center in the actual $1 \mathrm{ML}$ band. The surface electron energy per unit area in each case is given by

$$
E_{\mathrm{SS}}=\int^{E_{F}} \epsilon D(\epsilon) d \epsilon \text {. }
$$

$D(\epsilon)$ accounts for the density of states, which is defined as

$$
D(\epsilon)=2 \times \frac{1}{(2 \pi)^{2}} \int_{E(k)=\text { const }} \frac{d S_{E}}{\left|\operatorname{grad}_{k} E(k)\right|},
$$




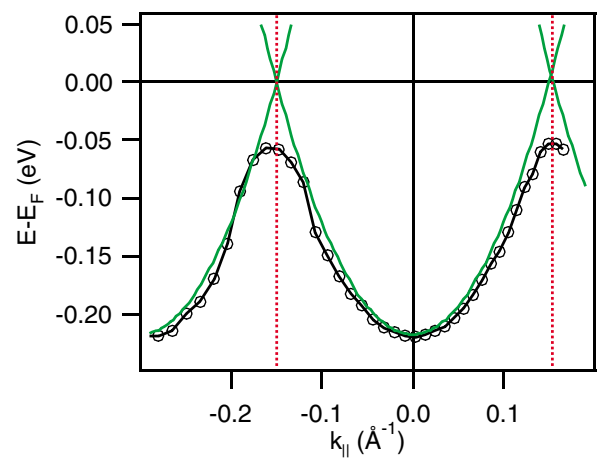

FIG. 4 (color online). Surface band for the 1 ML Ag film (data points) and the same band without the moiré superstructure potential (solid line). Since only a minor fraction of electrons at the zone boundary change their energy, the $65 \mathrm{meV}$ gap up to the Fermi level leads to a surface energy gain of $0.4 \mathrm{meV} /$ atom.

where $d S_{E}$ is an area element of the constant energy surface perpendicular to $k$. Equations (1) and (2) are numerically integrated for the measured 1 ML band of Fig. 4 and assuming isotropic dispersion in the surface plane. This results in $E_{\mathrm{SS}}=2.4 \mathrm{meV}$ per $\mathrm{Ag}$ surface atom. In contrast, the energy for a free-electron band with the same effective mass is $E_{\mathrm{SS}}=2.8 \mathrm{meV} /$ atom. Thus, there is a $0.4 \mathrm{meV} /$ atom gain by opening the gap at the zone boundary, very close to the previous estimate of the elastic cost $\Delta U$.

Figure 4 strongly suggests that the $\sim(9.5 \times 9.5) \mathrm{Ag}$ monolayer represents a $2 \mathrm{D}$ charge density wave (CDW) state. It describes a perfect nesting along the [110] directions of the surface, with the wave vector of the Ag monolayer band equaling half of the $(9.5 \times 9.5)$ superstructure lattice constant. On the other hand, there is full occupation of the surface band for a $(9.5 \times 9.5)$ reconstruction. The total charge carried by the surface state is calculated by integrating the density of states up to the Fermi energy. It is the same for the two bands in Fig. 4 (with or without gap), and hence there is no need to know the detailed Fermi surface of the actual $1 \mathrm{ML}$ band. For a free-electron-like surface state, Eq. (2) gives the constant value of $D(\epsilon)=$ $m^{*} / 2 \pi \hbar^{2}$. The charge carried by the 1 ML band in Fig. 4 is estimated as $n_{\mathrm{Ag}, \mathrm{ML}}=D(\epsilon) \times E_{\mathrm{Ag}, \mathrm{ML}}=0.021$ per Cu surface atom, which directly converts into $n_{e}^{G}=1.9$ electrons for a $(9.5 \times 9.5)$ unit cell. Given the uncertainty in effective mass and surface state energy, this number indicates full band occupation.

An upper estimate of the transition temperature $T_{\mathrm{CDW}}$ for the CDW state can be obtained from the gap value $\Delta$ and using the mean field theory formula $\Delta=3.5 k_{B} T_{c}$. In the present case $\Delta=86 \mathrm{meV}$ gap and hence $T_{\mathrm{CDW}}=$ $295 \mathrm{~K}$. However, first measurements taken at $300 \mathrm{~K}$ do not show remarkable differences in the gap value, suggesting that the CDW could be pinned in the present $(\sim 9.5 \times$ 9.5) triangular structure.
In summary, we have observed a zone-boundary gap at the Fermi energy for $1 \mathrm{ML} \mathrm{Ag}$ on $\mathrm{Cu}(111)$, which could explain the incommensurate $\sim(9.5 \times 9.5)$ moire superstructure that characterizes this system. Such a gap leads to an electron energy gain which is estimated to be sufficient to balance the elastic energy required to modify nearby commensurate reconstructions. Our analysis suggests that this incommensurate $\sim(9.5 \times 9.5)$ moiré represents a charge density wave state, which encourages further temperature-dependent Fermi surface measurements.

We acknowledge support from the DAAD/MCyT interchange program and the EC 6th framework Network of Excellence NANOQUANTA (NMP4-CT-2004-500198). J.E. O. and J. C. are funded by the Spanish Ministerio de Ciencia y Tecnología (MAT2002-03427, MAT-200211975-E, HA2002-0107) and the Universidad del País Vasco (1/UPV/EHU/00057.240-EA-13668/2001). Fruitful discussions with F. J. Himpsel, M. A. Cazalilla, and E. G. Michel are gratefully acknowledged.

[1] A. Damascelli, Z. Hussain, and Z.-X. Shen, Rev. Mod. Phys. 75, 473 (2003).

[2] R.E. Peierls, Quantum Theory of Solids (Oxford University Press, New York, 1955); G. Grüner, Density Waves in Solids (Addison-Wesley Publishing Company, Reading, MA, 1994).

[3] J. Schäfer et al., Phys. Rev. Lett. 83, 2069 (1999); J. Voit et al., Science 290, 501 (2000); K. Swamy et al., Phys. Rev. Lett. 86, 1299 (2001); J. R. Ahn et al., Phys. Rev. Lett. 93, 106401 (2004).

[4] T. Nakagawa et al., Phys. Rev. Lett. 86, 854 (2001).

[5] F. Besenbacher, L. P. Nielsen, and P. T. Sprunger, The Chemical Physics of Solid Surfaces and Heterogeneous Catalysis (Elsevier, Amsterdam, 1997), Vol. 8, Chap. 6.

[6] B. Aufray et al., Microsc. Microanal. Microstruct. 8, 167 (1997).

[7] I. Meunier et al., Phys. Rev. B 59, 10910 (1999).

[8] For the sake of simplicity, we disregard the vertical relaxation of the $\operatorname{Ag}(111)$ layer on $\mathrm{Cu}(111)$, i.e., the moiré corrugation which effectively reduces the compression in all reconstructions.

[9] A. Bendounan et al., Surf. Sci. 496, L43 (2002); A. Bendounan et al., Phys. Rev. B 67, 165412 (2003).

[10] T. Greber, T. J. Kreutz, and J. Osterwalder, Phys. Rev. Lett. 79, 4465 (1997).

[11] The normalization using the Fermi-Dirac distribution function is used only to qualitatively prove the existence of features above $E_{F}$, but must not be performed before the peak fit is done; see F. Schiller et al., Phys. Rev. B 70, 174444 (2004).

[12] J. Xie et al., Phys. Rev. B 59, 965 (1999).

[13] C. Mottet, G. Tréglia, and B. Legrand, Phys. Rev. B 46, 16018 (1992).

[14] C. Kittel, Introduction to Solid State Physics (John Wiley and Sons, New York, 1985), 10th ed.

[15] A further possibility to estimate $\Delta U$ is the use of the $\mathrm{Ag}$ bulk compression module $K=1.007 \times 10^{-11} \mathrm{~m}^{2} / \mathrm{N}$ and $U=\frac{1}{2} K \delta^{2}[14]$ resulting in $\Delta U=0.56 \mathrm{meV}$. 